\title{
Analysis of opto-thermal interaction of porcine stomach tissue with 808-nm laser for endoscopic submucosal dissection
}

\author{
Seongjun $\operatorname{Kim}^{\dagger} \star \star$ \\ Department of Mechanical Engineering \\ Graduate School, Kookmin University \\ Seoul 136-702, Republic of Korea \\ M. Tamim Hossain* \\ Department of Integrative Biomedical Science \\ and Engineering, Graduate School, Kookmin University \\ Seoul 136-702, Republic of Korea \\ Don Haeng Lee \\ Division of Gastroenterology \\ Department of Internal Medicine \\ Inha University School of Medicine \\ National Center of Efficacy Evaluation for the Development \\ of Health Products Targeting Digestive Disorders \\ Utah-Inha DDS \& Advanced Therapeutics Research Center \\ 27 Inhang-ro, Jung-gu, Incheon 400-711, Republic of Korea \\ Jung Kyung Kim* \\ Department of Mechanical Engineering \\ Kookmin University \\ Seoul 136-702, Republic of Korea \\ *jkkim@kookmin.ac.kr
}

Received 8 April 2015

Accepted 26 August 2015

Published 7 October 2015

\begin{abstract}
In endoscopic submucosal dissection (ESD), the narrow gastrointestinal space can cause difficulty in surgical interventions. Tissue ablation apparatuses with high-power $\mathrm{CO}_{2}$ lasers or $\mathrm{Nd}$ :YAG lasers have been developed to facilitate endoscopic surgical procedures. We studied the
\end{abstract}

\footnotetext{
${ }^{*}$ Corresponding author.

$\dagger$ Current affiliation: Asan Medical Center, 88, Olympic-ro 43-gil, Songpa-gu, Seoul 05505, Republic of Korea.

\$These two authors contributed equally to this study.
}

This is an Open Access article published by World Scientific Publishing Company. It is distributed under the terms of the Creative Commons Attribution 3.0 (CC-BY) License. Further distribution of this work is permitted, provided the original work is properly cited. 


\title{
S. Kim et al.
}

\begin{abstract}
interaction of 808-nm laser light with a porcine stomach tissue, with the aim of developing a therapeutic medical device that can remove lesions at the gastrointestinal wall by irradiating a near-infrared laser light incorporated in an endoscopic system. The perforation depths at the porcine fillet and the stomach tissues linearly increased in the range of $2-8 \mathrm{~mm}$ in proportion to the laser energy density of $63.7-382 \mathrm{~kJ} / \mathrm{cm}^{2}$. Despite the distinct structural and compositional difference, the variation of the perforation depth between the stomach and the fillet was not found at 808 -nm wavelength in our measurement. We further studied the laser-tissue interaction by changing the concentration of the methyl blue solution used conventionally as a submucosal fluidic cushion (SFC) in ESD procedures. The temperature of the mucosal layer increased more rapidly at higher concentration of the methyl blue solution, because of enhanced light absorption at the SFC layer. The insertion of the SFC would protect the muscle layer from thermal damage. We confirmed that more effective laser treatment should be enabled by tuning the opto-thermal properties of the SFC. This study can contribute to the optimization of the driving parameters for laser incision techniques as an alternative to conventional surgical interventions.
\end{abstract}

Keywords: Near-infrared laser; surgical intervention; laser-tissue interaction; submucosal fluid cushion; temperature distribution.

\section{Introduction}

The two prevalent methods for removing cancerous or other abnormal lesions from the gastrointestinal wall are endoscopic mucosal resection (EMR) and endoscopic submucosal dissection (ESD). ${ }^{1}$ EMR functions by using the endoscopic channel to locate a snare around the lesion, then removing the lesion using a high-frequency electric current. Unfortunately, EMR has limits regarding lesion size, which introduces difficulties for the surgeon. ESD has overcome this disadvantage, improving accuracy of the procedure by removing the whole lesion at once. However, ESD's long performance time, difficulty, and increased resulting bleeding and perforations are disadvantageous. Both the removal methods based on endoscopy use tools such as snares and needle knives, which can be difficult to operate within the narrow space of the digestive system owing to restricted range of motion and hence lead to bleeding and perforations at the time of operation. ${ }^{2,3}$ For these reasons, the development of a device to remove malignant or benign tumor, that uses a high-powered laser for simultaneous incision and hemostasis through optical fibers is necessary.

Lasers have many applications in medical fields. For tissue ablation in surgical procedures, different types of laser have been used previously such as potassium titanyl phosphate (KTP), neodymiumdoped yttrium aluminium garnet (Nd:YAG) and $\mathrm{CO}_{2}$ laser. The most widely used variety is $\mathrm{CO}_{2}$ gas laser which has a wavelength of $9.4-10.6 \mu \mathrm{m}$. $\mathrm{Au}$ et $a l_{.}{ }^{4}$ showed that irradiation with $\mathrm{CO}_{2}$ laser can cut lesions and maintain hemostasis simultaneously because the target tissue's high absorption protects the surrounding tissues from damage and the laser creates a clear cut surface. Also, it has advantage of better visibility due to low level of hemorrhaging. However, performing ESD using a $\mathrm{CO}_{2}$ laser can be troublesome because its structure limits movement. ${ }^{5,6}$ High absorption in water and protein with higher wavelength $(10.6 \mu \mathrm{m})$ prevents it from penetrating deep tissues. ${ }^{7,8}$ Our previous study $^{9}$ showed that infrared laser light within the 800-1100 nm wavelength band shows low absorption in hemoglobin, melanin, protein, and water. For this reason, we can deliver energy to deep tissues in an easy-to-control manner using flexible optical fibers with $808-\mathrm{nm}$ wavelength of laser. ${ }^{10}$ A serious side effect in the removal of tumors using both EMR and ESD is the perforation of the gastrointestinal wall. Fujishiro et al. ${ }^{11}$ demonstrated that the most effective protection against perforation is the insertion of a submucosal fluidic cushion (SFC), which creates space between the mucosal and submucosal layers. SFC solution is commonly composed of distilled water, physiological saline, glycerin, hyaluronic acid, and other additives. ${ }^{12-15}$ The SFC can lift the lesion from the submucosal layer and thus protect nontarget tissues from bleeding and perforation.

As mentioned above, the most serious side effects of ESD are perforation and post-operative bleeding. With laser technology, surgeons can accomplish ablation and hemostasis at the same time, which 
prevents bleeding. The insertion of a SFC under the mucosal layer can prevent perforation of the digestive wall. However, laser irradiation can cause thermal damage to surrounding tissues. Thermal damage of nontarget tissue can be prevented using suitable driving conditions that consider the thermal diffusivity of the tissue. In this study, we performed a preliminary research that may guide the invention of an ESD device using 808-nm high-power infrared laser light. We studied the effect of laser driving parameters, such as irradiation time and output power, and the type of tissue on the perforation depth. We also measured the depthwise temperature distribution in the cross-section of the porcine tissue sample when a SFC gel was inserted under the mucosal layer to prevent perforation. Finally, we measured the laser absorption coefficient of methyl blue in the SFC gel in accordance with its concentration. Based on the measured absorption coefficient, we examined the temperature distribution in the extracted porcine stomach tissue as affected by the concentration of methyl blue in the SFC.

\section{Materials and Methods}

\subsection{Experimental apparatus}

We used a high-power near-infrared InGaAs diode laser (Passive Cooled Ocla ${ }^{\text {TM }}$ Laser, NDLUX) with an emitted wavelength of $808 \mathrm{~nm}$. The output power range of the device is $1-15 \mathrm{~W}$ and the frequency range is between 1 and $600 \mathrm{~Hz}$. It can also be set to emit a continuous wave. Laser light was irradiated to the sample through an optical fiber with a diameter of $400 \mu \mathrm{m}$ and a length of $2.5 \mathrm{~m}$, held on a two-axis stage that can be moved in both $x$ and $y$ directions. The optical fiber was in contact only with the flattened sample surface. The size of the beam spot formed at the sample surface was about $1 \mathrm{~mm}$. The temperature distribution at the cross-section of the tissue sample was measured using a thermal imaging camera $\left(\right.$ MoblR $^{\circledR}$ M8, NewTech Instruments). Thermal imaging cameras are noninvasive devices that can measure the temperature at a surface by detecting and quantifying emitted infrared radiation. ${ }^{16}$ The recorded thermal images were analyzed using the Guide IrAnalyser program. Figures 1(a) and 1(b) show the experimental setups for laser irradiation and temperature measurement, respectively. Fresh porcine fillet and stomach tissues were obtained by a local slaughter

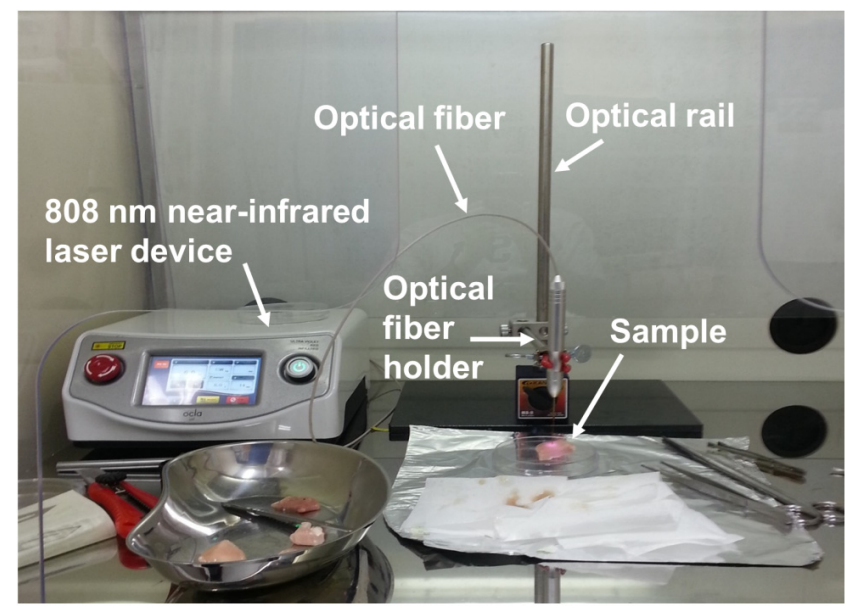

(a)

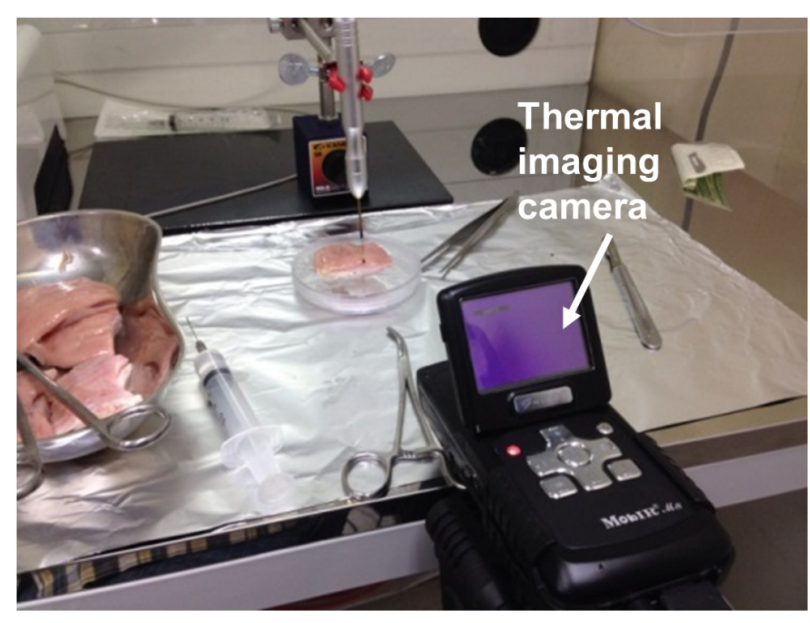

(b)

Fig. 1. (a) Experimental setup showing the 808-nm infrared laser device and the irradiation of porcine tissue sample with an optical fiber, an optical fiber holder and a vertically movable optical rail. (b) Thermal imaging setup for measurement of the cross-sectional temperature distribution in the porcine stomach tissue.

house and used as test samples. The organs were collected from different animals and experiments were performed at the same day after collecting the organs.

\subsection{Measurement of perforation depth}

The perforation depth measurement, as affected by laser driving parameters such as output power and irradiation time, was executed with regularly sized fillet and stomach tissue samples. The samples were irradiated using combinations of different output powers $(5-15 \mathrm{~W})$ and irradiation times (5-60 s). 


\section{S. Kim et al.}

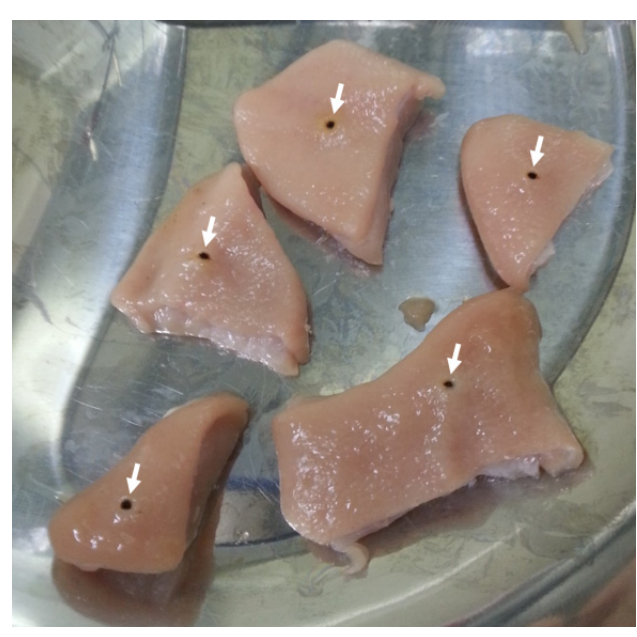

(a)

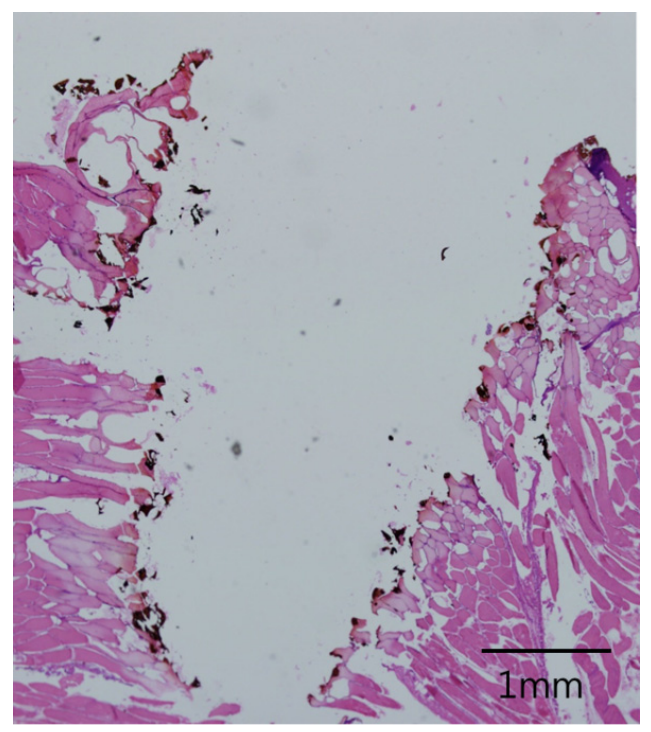

(c)

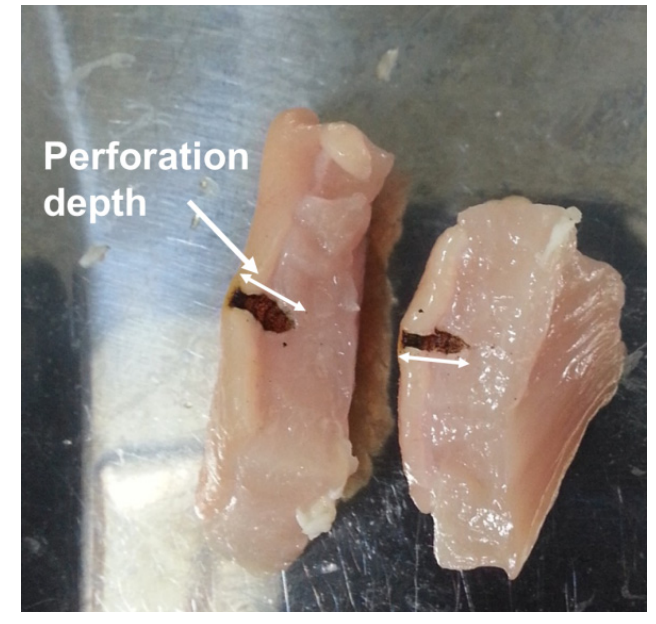

(b)

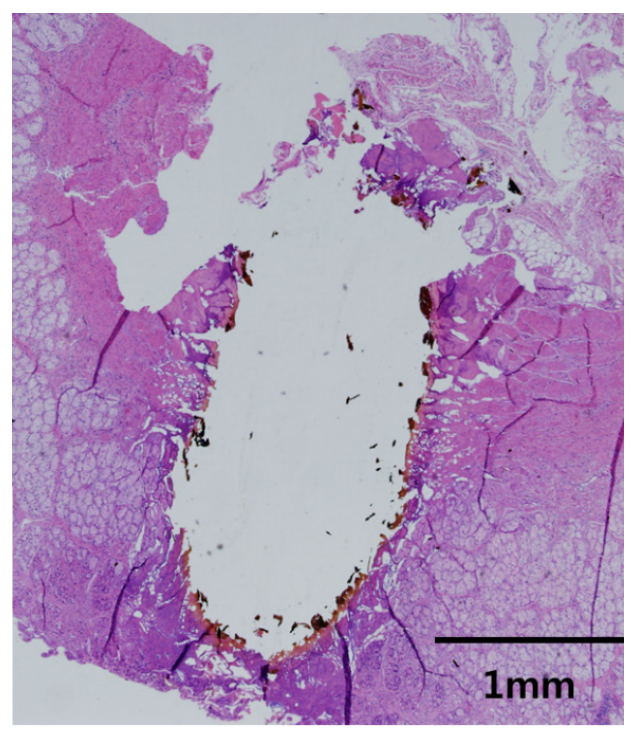

(d)

Fig. 2. (a) Perforated porcine tissue samples after laser irradiation. The black dot (indicated with a white arrow) in each tissue sample is the carbonized spot formed at the superficial layer. (b) Measurement of the perforation depth at the cross-section of the tissue sample. Microscopic hematoxylin and eosin-stained cross-sections of the (c) porcine fillet and (d) porcine stomach tissues.

Figure 2(a) displays the perforated fillet tissue samples after irradiation. We measured the perforation depth using Vernier calipers after the perforation hole was cut vertically as shown in Fig. 2(b). The measurement of perforation depth was based on the carbonization area of the surface that had been cut. To obtain statistical data, we repeated each test five times and took the average depth measurement. The irradiated samples were fixed in $10 \%$ formalin and embedded in paraffin for hematoxylin and eosin $(\mathrm{H} \& \mathrm{E})$ staining for studying histology. $\mathrm{H} \& \mathrm{E}$ stained tissue samples are shown in
Figs. 2(c) and 2(d) for the perforated fillet and the stomach tissues, respectively.

\subsection{Effect of SFC on laser ablation in porcine tissue-agar gel model}

In order to replicate the effect of a SFC in ESDbased endoscopy, we used thin porcine fillet samples to shape a multi-layered structure as shown in Fig. 3. The average thickness of the porcine sample was $2.14 \mathrm{~mm}$. A semi-solid agar gel of $1 \%$ concentration $(\mathrm{w} / \mathrm{v})$ was used as a SFC material. Even 


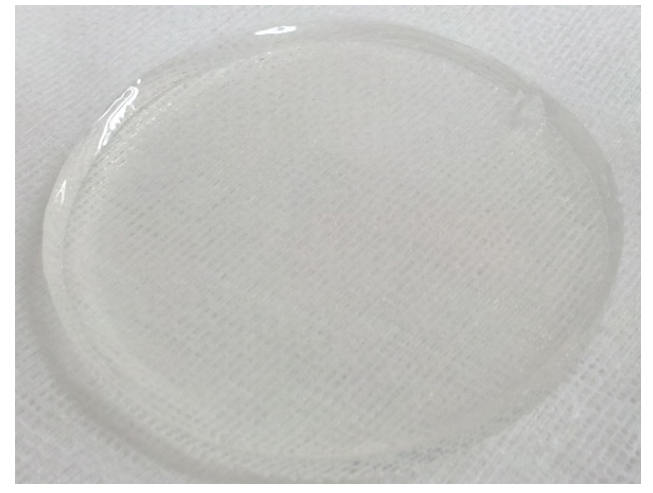

(a)

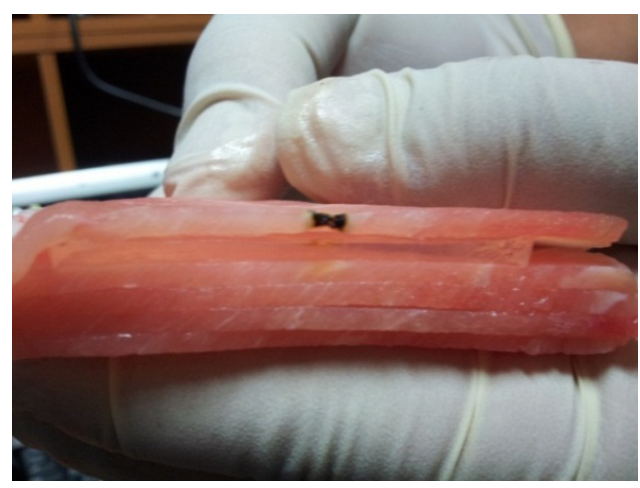

(c)

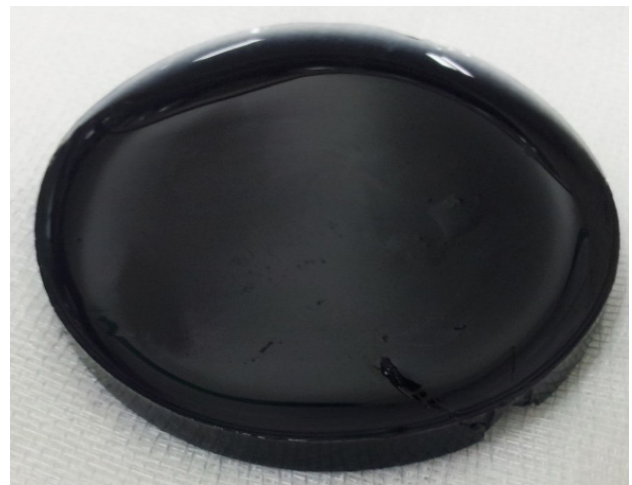

(b)

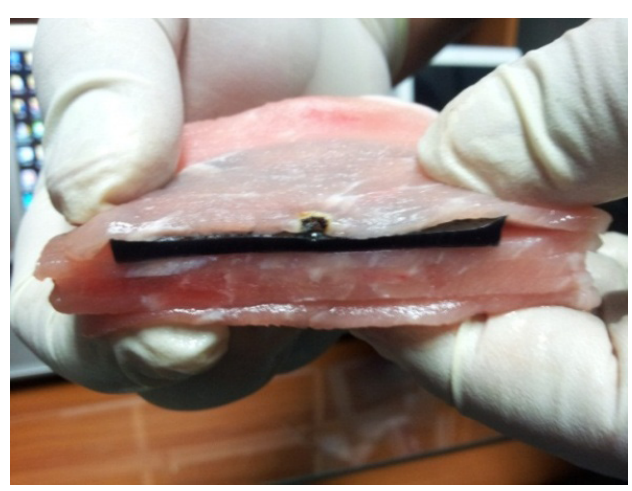

(d)

Fig. 3. Multi-layered porcine tissue-agar gel model. Agar gel of $1 \%$ concentration was used as a SFC material. (a) Normal gel, (b) black gel, (c) tissue sample with normal gel under the top layer after laser irradiation, and (d) tissue sample with black gel under the top layer after laser irradiation.

though hypertonic saline solution has been used as a SFC in the medical field, we used a hydrogel to facilitate the measurement of the temperature at the cross-section of the tissue sample using a thermal imaging camera. The time course of the temperature rise was measured in the tissue sample with the insertion of a colorless normal gel [Figs. 3(a) and $3(\mathrm{c})]$ or a black gel [Figs. 3(b) and 3(d)]. The thickness of the gel was $3 \mathrm{~mm}$. We ensured that both the porcine sample and the gel were allowed to reach the same temperature before applying laser irradiation. The optical fiber was placed at the distal end of the cut surface, which was then irradiated with laser light at $10 \mathrm{~W}$ for $30 \mathrm{~s}$ in a direction normal to the top layer of the tissue sample. The thermal imaging camera was located perpendicular to the cross-section of the sample to measure the depthwise temperature distribution. We monitored the temperatures at three different points: the top layer, the agar gel, and the middle layer. After laser irradiation, we measured the perforation depth in all three different cases of no gel, colorless gel, and black gel.

\subsection{Effect of SFC on temperature distribution in porcine stomach tissue}

In conventional treatment, the SFC is applied as a mixture of the hypertonic saline solution with the methyl blue, which allows for visual clarity during ESD procedures. In this study, due to the use of a laser, we attempted to increase the efficacy and safety of the treatment through the adjustment of the light absorption properties of the SFC by changing the concentration of the methyl blue solution. Before measuring the temperature distribution in the cross-section of the stomach tissue sample, the light absorption coefficient was measured according to the concentration of the methyl 


\section{S. Kim et al.}

blue in the SFC. Five different concentrations (0.5, $0.1,0.05,0.01$ and $0.001 \%$ ) of methyl blue solutions were used to determine the absorption coefficient at various incident laser output powers $(1,3$ and $5 \mathrm{~W})$. A thermal laser power sensor (12A, Ophir) and a laser power meter (NOVA II, Ophir) were employed to measure the reduction in the transmitted laser output corresponding to the concentration of methyl blue. The actual power output of each laser power setting was measured using distilled water without the addition of dye. The reduced output value as affected by the concentration of methyl blue was measured against the actual output value. Based on the output values measured in each condition, the absorption coefficient of the SFC based on the concentration of methyl blue solution was calculated using the Beer-Lambert law, $I=I_{0} \exp (-\alpha d)$, where $I_{0}$ and $I$ are an incident laser intensity (W) and a transmitted laser intensity (W) through the sample medium, respectively, $\alpha$ is a light absorption coefficient $\left(\mathrm{cm}^{-1}\right)$ and $d$ is a thickness $(\mathrm{cm})$ of the sample medium.

We monitored the temperatures at three different points; the mucosal layer, the SFC, and the submucosal layer in the stomach tissue sample. The SFC was inserted into the submucosal layer, and the temperature distribution within the cross-section was visualized using a thermal imaging camera during laser irradiation. The tested SFC was composed of saline $(0.9 \% \mathrm{NaCl})$ with a concentration of either $0.01 \%, 0.1 \%$ or $1 \%$ methyl blue. The mucosal layer rose after the injection of $5 \mathrm{~mL}$ of SFC using a syringe, and the cross-section appeared after the perforation was made at the center of the lifted surface. In the experiment, the tissue was irradiated for $30 \mathrm{~s}$ with the laser in a continuous wave mode at $5 \mathrm{~W}$ output.

\section{Results and Discussion}

\subsection{Perforation depth at different porcine organs}

Porcine fillet and digestive organs (in this case, the stomach) have different tissue characteristics, such as phlegmatic versus wrinkled surface structures. To determine the effect of tissue properties on lasertissue interaction, we measured the perforation depth using the same method for both types of the tissue samples. We confirmed the presence of carbonization and coagulation around perforation holes in both types. We measured the perforation depth at different output powers and irradiation times, repeated each condition five times, and calculated the average depth. We compared the results obtained in the fillet and stomach tissue samples under the same condition. The comparison of perforation depth at various total energy levels between 50 and $300 \mathrm{~J}$ is shown in Fig. 4.

As depicted in Figs. 4(a) and 4(b), the perforation depth data for both fillet and stomach tissue exhibit a linear rise with the increasing total applied energy. We used high irradiation time with low output power and low irradiation time with high output power to equalize the energy level. The laser output power did not affect the perforation depth at

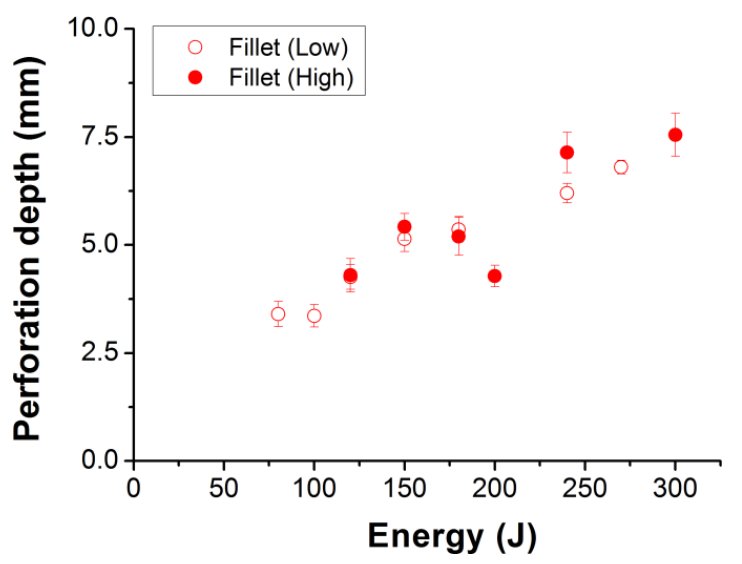

(a)

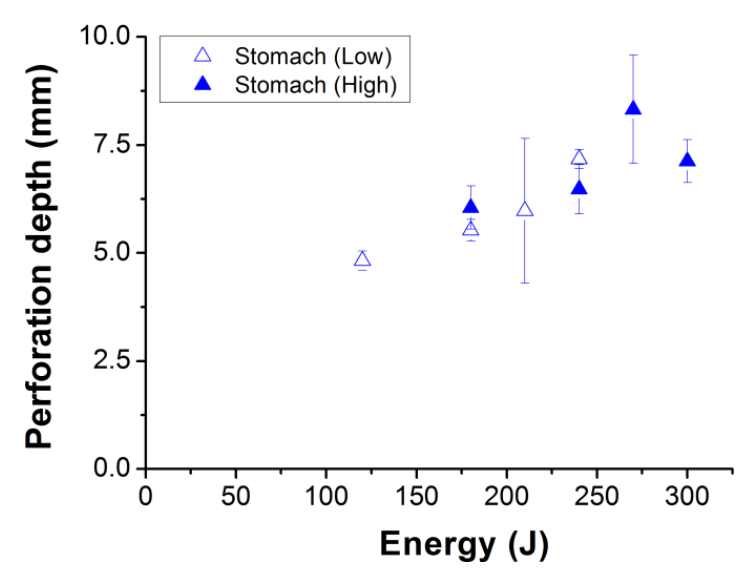

(b)

Fig. 4. Perforation depths (a) in the fillet and (b) in the stomach tissue samples at low $(<10 \mathrm{~W})$ and high $(\geq 10 \mathrm{~W})$ laser output powers. Comparison of perforation depth in the fillet and stomach tissue samples at (c) low and (d) high output powers. 


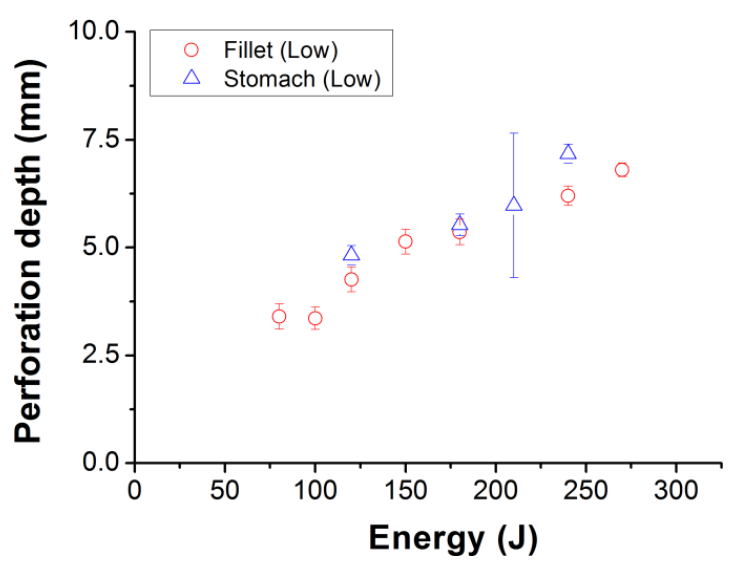

(c)

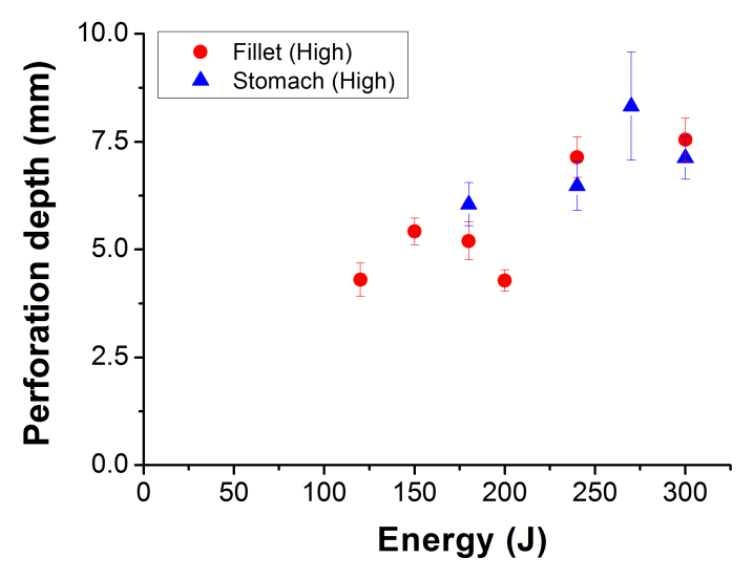

(d)

Fig. 4. (Continued)

the same energy level. No significant difference of perforation depth between fillet and stomach tissue was found as compared in Figs. 4(c) and 4(d) at both low $(<10 \mathrm{~W})$ and high $(\geq 10 \mathrm{~W})$ output powers, respectively. This observation implies that the output power and operational time can be varied to obtain desired energy during treatment. Initially, we hypothesized that the thin sticky layer at the porcine stomach would cause a difference in perforation depth by absorbing higher laser energy which increases temperature rapidly at the early stage. However, there is no significant difference between the stomach and the fillet, which is attributed to the characteristics of the wavelengths of $800-1100 \mathrm{~nm}$ that exhibit low absorption at hemoglobin, melanin, protein and water. An effective laser-assisted surgical technique for soft tissue ablation should be selected based on the wavelength of light. ${ }^{17}$ The penetration depth of 808 -nm light was measured to be larger than other wavelengths. ${ }^{18}$ Our perforation depth data obtained for the 808-nm laser at various applied energies would provide a general understanding of laser-tissue interaction in a digestive organ and aids in determining the laser driving conditions most optimal for incising stomach tissues.

\subsection{Temperature distribution in porcine tissue-agar gel model}

The perforation depth measurements using an agar gel that replicated SFC were performed after laser irradiation at $10 \mathrm{~W}$ output power for $30 \mathrm{~s}$. The experiment was performed for three different cases: no

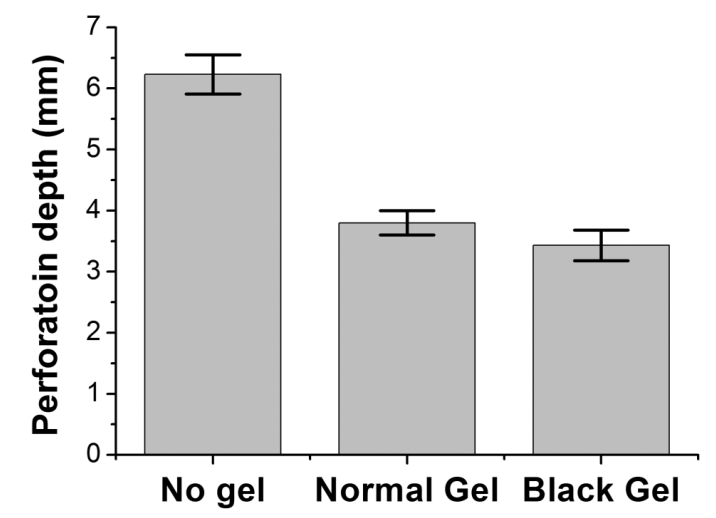

Fig. 5. Effect of agar gel insertion on ablation depth in multilayered porcine tissue-agar gel model.

gel, colorless gel, and black gel insertion. The average perforation depths were obtained from data collected in three repetitions of each case and displayed in Fig. 5. The difference in perforation depth based on the presence of the gel was large. We confirmed that the gel inserted between the top and middle layers prevented perforation at the middle layer of the tissue model. The perforation depth showed little difference based on the color of the gel.

The change in the temperature distribution at the cross-section of the tissue-agar gel model was measured at three different points: the top layer (point 1), the gel layer (point 2), and the middle layer under the gel (point 3) as illustrated in Fig. 6(a). Figure $6(\mathrm{~b})$ shows the depthwise distribution of the temperature rise in the case of no gel, where the points 2 and 3 exist at the middle and bottom layers, respectively. The temperature rises at point 1 and then at point 2 , but there is no considerable 


\section{S. Kim et al.}

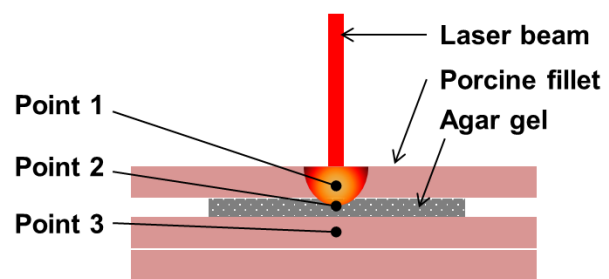

(a)

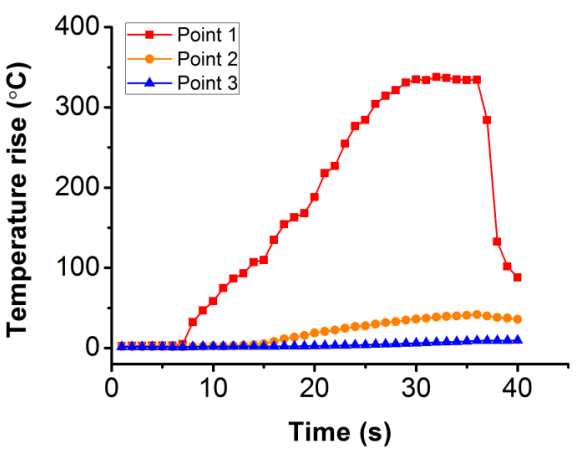

(c)

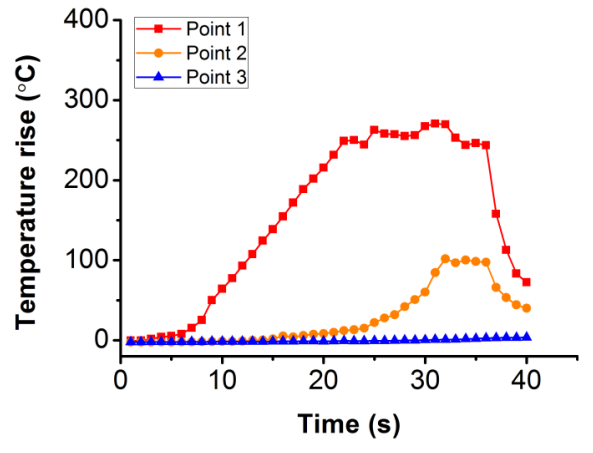

(b)

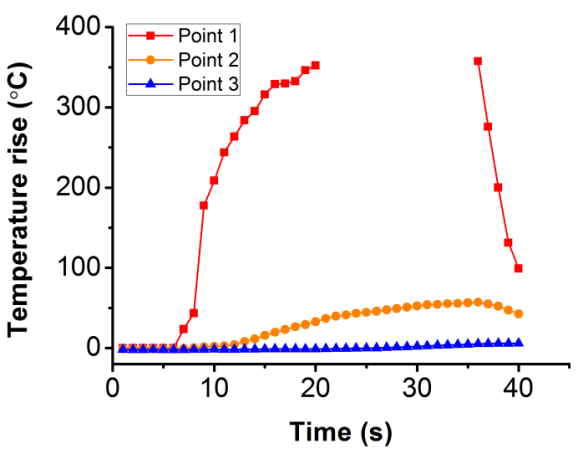

(d)

Fig. 6. Time course of temperature rise at different depths in multi-layered porcine tissue-agar gel model for each condition over the course of a 40-s laser irradiation at $10 \mathrm{~W}$. (a) Schematic of multi-layered porcine tissue-agar gel model and indications of the points for temperature measurements. (b) No gel, (c) colorless normal gel, and (d) black gel.

increase in temperature at point 3 over the course of irradiation. In the case of both colorless [Fig. 6(c)] and black [Fig. 6(d)] gel insertions, at point 1, the temperature increases much more swiftly than it has been with no gel present. However, the temperature increase at point 2 is smaller than it has been with no gel present. This is because the light absorption coefficient of the gel is smaller than that of porcine fillet. There was no difference at point 3 in any of the cases.

We also measured the effect of gel color on perforation depth, using $1 \%$ concentration of colorless and black gel at the middle layer. The perforation depth decreased when the gel was inserted, but the difference of perforation depth for black versus colorless gel was not significant. There were differences amongst the temperature distributions of the crosssection of the sample in all three cases. In the case of no gel, the temperature at point 1 was smaller compared with both cases that used a gel insertion. However, the temperature at point 2 was larger in the no-gel case than it was in other cases. The temperature increase at point 1 was larger in the case of gel insertion than other cases. The temperature increased to a great extent in the case of black gel and the increase above $350{ }^{\circ} \mathrm{C}$ could not be detected by the thermal imaging camera [Fig. 6(d)]. Point 3 showed no large difference in any of the three cases. In previous studies measuring the thermal conductivity and thermo-absorption of porcine tissue as affected by the concentration of agar gel, we confirmed a high thermo-absorption and low thermal conductivity in tissue; hence, when gel is used, the perforation depth decreases and the temperature increases. Therefore, the use of gel replicating a SFC is beneficial in preventing perforation and thermal diffusion within the tissue wall.

\subsection{Effect of SFC on laser-tissue interaction}

In this experiment, we used methyl blue as a SFC dye and measured the absorption coefficient of methyl blue solution at five concentrations $(0.5,0.1$, 


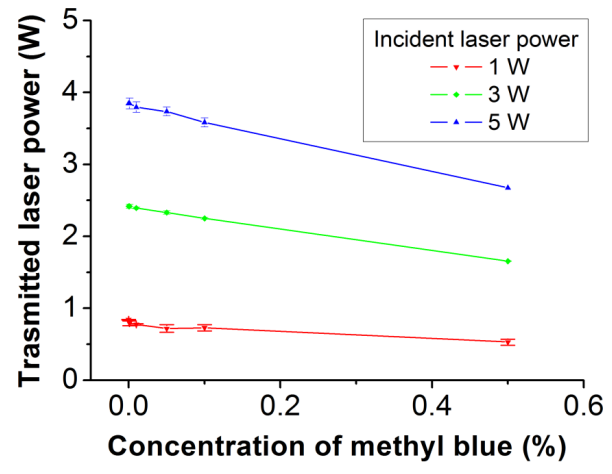

(a)

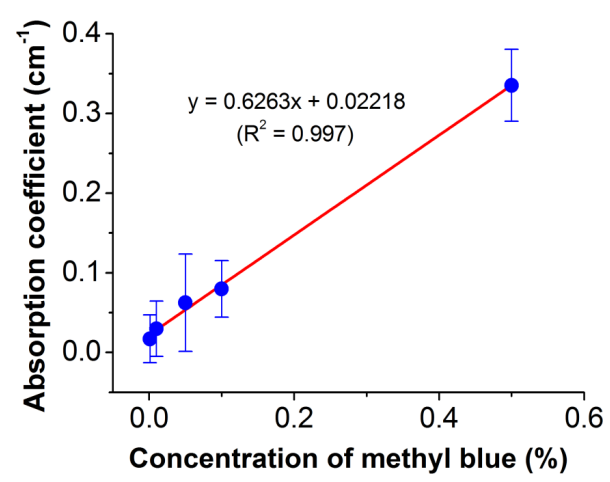

(b)

Fig. 7. (a) Transmitted laser power varied with different concentrations of methyl blue at 3 incident laser powers (1, 3 and $5 \mathrm{~W}$ ). (b) The absorption coefficients of the methyl blue solution determined by the Beer-Lambert law at different concentrations.

$0.05,0.01$, and $0.001 \%$ ) and three incident laser output powers (1, 3 and $5 \mathrm{~W})$. The measurement of the transmitted laser powers is shown in Fig. 7(a). We calculated the absorption coefficient of methyl blue based on the Beer-Lambert law using the measured values as shown in Fig. 7(b). The absorption of laser power increased linearly with the concentration of methyl blue, which implies that we can control the opto-thermal properties of a SFC through changing the concentration of methyl blue.

To find the effect of SFC on laser-tissue interaction, we irradiated the porcine stomach sample where the SFC (1\% methyl blue solution) was inserted in the submucosal layer at $5 \mathrm{~W}$ for $30 \mathrm{~s}$. The SFC protected the muscle layer in the stomach tissue from a carbonization as displayed in Fig. 8(b) compared with a stomach sample without SFC insertion in Fig. 8(a). To further study the effect of optical property of SFC on laser-tissue interaction, we measured the cross-sectional temperature

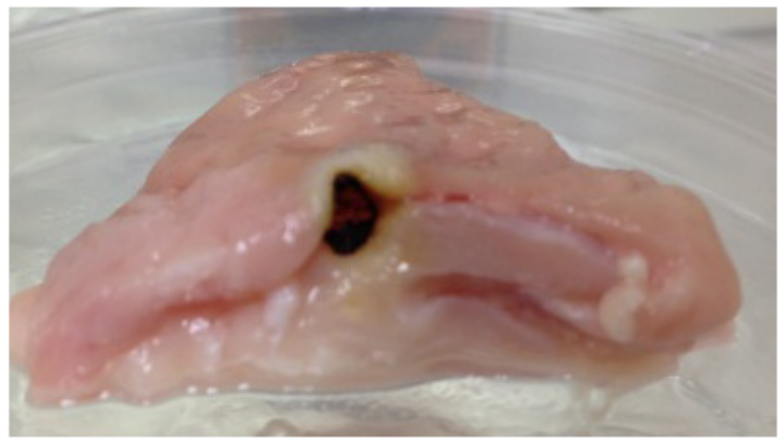

(a)

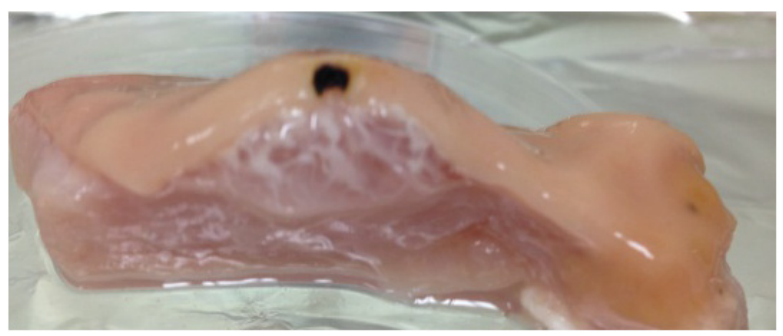

(b)

Fig. 8. Cross-sectional views of the perforated stomach tissue samples (a) without SFC insertion and (b) with SFC insertion after laser irradiation at $5 \mathrm{~W}$ for $30 \mathrm{~s}$. A methyl blue solution of $1 \%$ concentration was used for the SFC.

distribution at the porcine stomach sample according to the concentration of methyl blue solution.

Figure 9 shows the depthwise temperature profiles with increasing time at three different concentrations of methyl blue solutions, $0.01 \%$ [Fig. 9 (a)], $0.1 \%$ [Fig. 9(b)] and 1\% [Fig. 9(c)] during the early phase $(2-10 \mathrm{~s})$ of laser irradiation. The absorption coefficients of the $0.01 \%, 0.1 \%$ and $1 \%$ methyl blue were measured to be $0.0284,0.0848$ and $0.648 \mathrm{~cm}^{-1}$, respectively, in Fig. 7(b). At higher concentration, the temperature at the mucosal layer was increased more rapidly than lower concentration because the light absorption was enhanced at the SFC layer. Although the temperature rise for $6 \mathrm{~s}$ was negligible at $0.01 \%$ and $0.1 \%$ of methyl blue solutions [Figs. 9(a) and 9(b)], the temperature at $1 \%$ of methyl blue increased abruptly in the mucosal layer [Fig. 9(c)]. The absorbed light at the SFC layer is converted into heat, which transfers to the mucosal layer and increases its temperature. The rapid temperature rise above $100^{\circ} \mathrm{C}$ in the focused laser spot formed a perforation at the mucosal layer more quickly, but the temperature at the submucosal layer remained unchanged preventing 


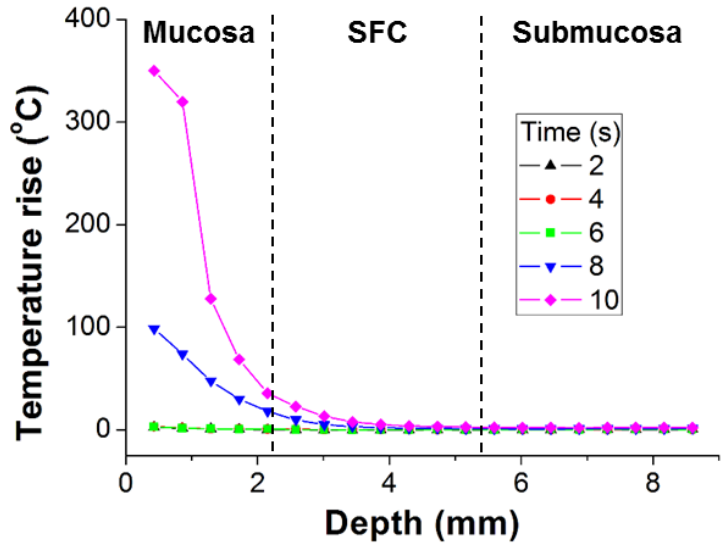

(a)

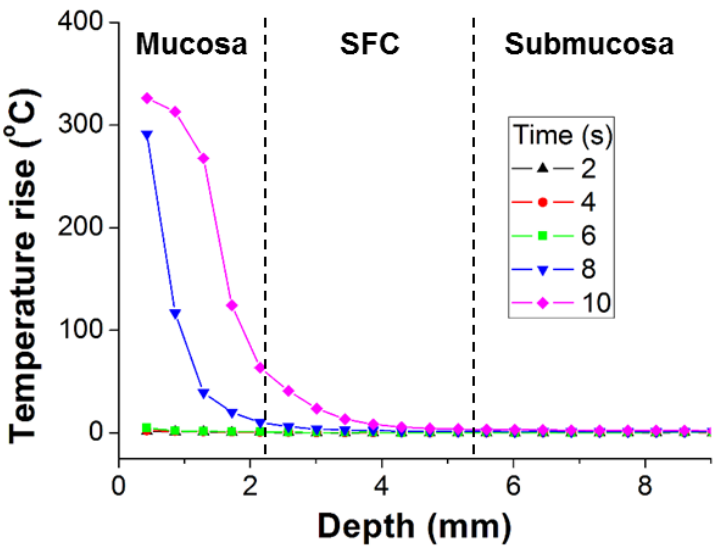

(b)

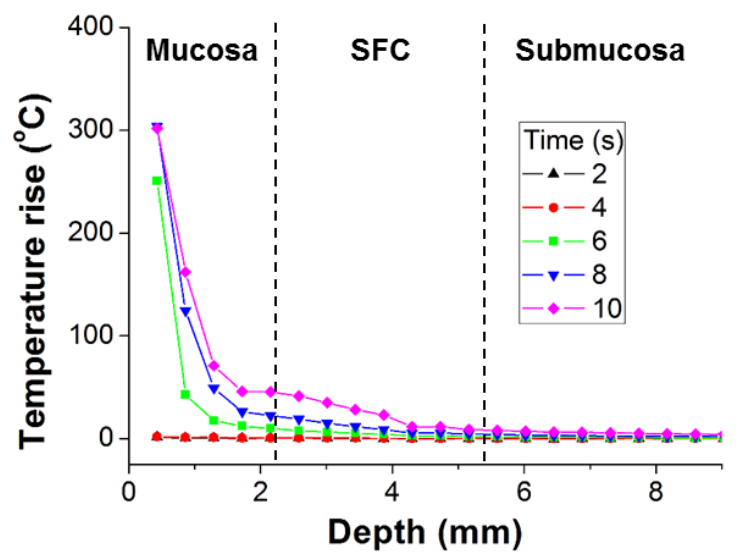

(c)

Fig. 9. Effect of SFC on temperature rise in porcine stomach tissue sample with laser irradiation at $5 \mathrm{~W}$ for $30 \mathrm{~s}$. Depthwise temperature profiles with increasing time at three different concentrations of methyl blue solution. (a) $0.01 \%$, (b) $0.1 \%$ and (c) $1 \%$.

muscular damages such as thermal injury and perforation, as demonstrated in Fig. 8(b). The time and energy required for a gastrointestinal endoscopic surgery using a laser can be reduced by tuning the opto-thermal properties of the SFC. ${ }^{19}$

\section{Conclusion}

We conducted a basic research on laser-tissue interaction to support the development of an 808-nm high-power near-infrared laser incision device for use in procedures such as ESD carried out in narrow spaces. We found that the perforation depth increased linearly with laser output power and irradiation time in both fillet and stomach tissue samples. The structural and compositional distinction between those two tissue types did not make any significant difference in perforation depth.
Based on this finding, we envisage that the 808-nm laser light can be used with controlled output power and irradiation time to obtain a desired perforation depth, which would provide a medical personnel with greater flexibility and control during treatment. We also measured the temperature distribution at the cross-section of the porcine stomach tissue when a methyl blue solution was injected into the submucosal layer as a SFC. We found that enhanced 808-nm light absorption by high-concentration methyl blue solution resulted in rapid temperature increase at the mucosal layer while maintaining the temperature constant at the submucosal and muscular layers. We conclude that a SFC with optimized opto-thermal properties can absorb the energy selectively and minimize complications at the non-lesional tissue by enhancing the light-tissue interaction. 


\section{Acknowledgment}

This study was supported by grants from the Industrial Core Technology Development Program (10047904) and the Industrial Strategic Technology Development Program (10049743) of the Korea Evaluation Institute of Industrial Technology (KEIT), and the Human Resources Development Program (20134010200580) of the Korea Institute of Energy Technology Evaluation and Planning (KETEP) funded by the Ministry of Trade, Industry and Energy, Republic of Korea. M. Tamim Hossain was funded by the Global Scholarship Program for Foreign Graduate Students at Kookmin University in Korea.

\section{References}

1. S. J. Park, "Possibility of endoscopic surgery," Kor. J. Intern. Med. 79, 455-458 (2010).

2. D. S. Han, "Gastrointestinal endoscopic surgery," Hanyang Med. Rev. 29(2), 75-82 (2008).

3. M. Karita, M. Tada, K. Okita, "The successive strip biopsy partial resection technique for large early gastric and colon cancers," Gastrointest. Endosc. 38(2), 174-178 (1992).

4. J. T. Au, A. Mittra, J. Wong, S. Carpenter, J. Carson, D. Haddad, S. Monette, P. Ezell, S. Patel, Y. Fong, "Flexible $\mathrm{CO}_{2}$ laser and submucosal gel injection for safe endoluminal resection in the intestines," Surg. Endosc. 26(1), 47-52 (2012).

5. S. E. Fisher, J. W. Frame, R. M. Browne, R. M. Tranter, "A comparative histological study of wound healing following $\mathrm{CO}_{2}$ laser and conventional surgical excision of canine buccal mucosa," Arch. Oral Biol. 28(4), 287-291 (1983).

6. J. R. Tuffin, J. A. Carruth, "The carbon dioxide surgical laser," Br. Dent. J. 149(9), 255-258 (1980).

7. J.-H. Cho, J. Y. Cho, M.-Y. Kim, S. R. Jeon, T. H. Lee, H. G. Kim, S. Y. Jin, S. J. Hong, "Endoscopic submucosal dissection using a thulium laser: Preliminary results of a new method for treatment of gastric epithelial neoplasia," Endoscopy 45(9), 725-728 (2013).

8. J. I. Youn, J. D. Holcomb, "Ablation efficiency and relative thermal confinement measurements using wavelengths $1,064,1,320$, and 1,444 $\mathrm{nm}$ for laserassisted lipolysis," Lasers Med. Sci. 28(2), 519-527 (2013).

9. J. Cho, H. Byun, S. Lee, J. K. Kim, "Temperature distribution in deep tissue phantom during laser irradiation at 1,064 nm measured by thermocouples and thermal imaging technique," J. Vis. 14(3), 265272 (2011).

10. M. N. Lee, S. H. Lee, C. C. Kim, "Clinical application of argon laser in pediatric dentistry," J. Kor. Acad. Pediatr. Dent. 24(1), 139-147 (1997).

11. M. Fujishiro, N. Yahagi, K. Kashimura, Y. Mizushima, M. Oka, S. Enomoto, N. Kakushima, K. Kobayashi, T. Hashimoto, M. Iguchi, Y. Shimizu, M. Ichinose, M. Omata, "Comparison of various submucosal injection solutions for maintaining mucosal elevation during endoscopic mucosal resection," Endoscopy 36(7), 579-583 (2004).

12. H. Yamamoto, H. Kawata, K. Sunada, K. Satoh, Y. Kaneco, K. Ido, K. Sugano, "Success rate of curative endoscopic mucosal resection with circumferential mucosal incision assisted by submucosal injection of sodium hyaluronate," Gastrointest. Endosc. 56, 507-512 (2002).

13. T. Uraoka, T. Fujii, Y. Saito, T. Sumiyoshi, F. Emura, P. Bhandari, T. Matsuda, K. Fu, D. Saito, "Effectiveness of glycerol as a submucosal injection for EMR," Gastrointest. Endosc. 61, 736-740 (2000).

14. A. B. Feitoza, C. J. Gostout, L. J. Burgart, A. Burkert, L. J. Herman, E. Rajan, "Hydroxypropyl methylcellulose: A better submucosal fluid cushion for endoscopic mucosal resection," Gastrointest. Endosc. 57, 41-47 (2003).

15. I. Kumano, M. Ishihara, S. Nakamura, S. Kishimoto, M. Fujita, H. Hattori, T. Horio, Y. Tanaka, K. Hase, T. Maehara, "Endoscopic submucosal dissection for pig esophagus by using photocrosslinkable chitosan hydrogel as submucosal fluid cushion," Gastrointest. Endosc. 75(4), 841-848 (2012).

16. M. Mital, E. P. Scott, "Thermal detection of embedded tumors using infrared imaging," J. Biomech. Eng. 129(1), 33-39 (2007).

17. H. W. Kang, J. Kim, J. Oh, "Effect of wavelength on laser-assisted surgical techniques," Opt. Laser. Eng. 51(2), 104-110 (2013).

18. D. E. Hudson, D. O. Hudson, J. M. Wininger, B. D. Richardson, "Penetration of laser light at 808 and $980 \mathrm{~nm}$ in bovine tissue samples," Photomed. Laser Surg. 31(4), 163-169 (2013).

19. D. Obata, Y. Morita, R. Kawaguchi, K. Ishii, H. Hazama, K. Awazu, H. Kutsumi, T. Azuma, "Endoscopic submucosal dissection using a carbon dioxide laser with submucosally injected laser absorber solution (porcine model)," Surg. Endosc. 27(11), 4241-4249 (2013). 